\title{
Sampling-Free Enforcement of Non-Gaussian Chance Constraints via Fourier Transforms*
}

\author{
Abraham P. Vinod \\ University of Texas at Austin \\ Austin, Texas, USA \\ aby.vinod@gmail.com
}

\author{
Vignesh Sivaramakrishnan \\ University of New Mexico \\ Albuquerque, New Mexico, USA \\ vigsiv@unm.edu
}

\author{
Meeko M. K. Oishi ${ }^{\dagger}$ \\ University of New Mexico \\ Albuquerque, New Mexico, USA \\ oishi@unm.edu
}

\begin{abstract}
Verification of stochastic dynamical systems can often be formulated as chance-constrained optimization problems - maximize probability of satisfaction of safety/reachability objectives subject to dynamics and control bounds. For linear systems perturbed by Gaussian noise, chance-constraint techniques have proven to be highly efficient. With the goal of extending this approach to nonGaussian disturbances, this short paper focuses on tractable approaches to enforce chance constraints involving non-Gaussian random vectors. After reviewing existing techniques, we propose a novel approach to enforce chance constraints for arbitrary disturbances using Fourier transforms that is sampling-free and provides tight approximations. We demonstrate the efficiency of our approach in a simple example.
\end{abstract}

\section{CCS CONCEPTS}

- Theory of computation $\rightarrow$ Stochastic control and optimization; Convex optimization; • Computing methodologies $\rightarrow$ Control methods; Computational control theory;

\section{INTRODUCTION}

In real world applications, uncertainties may arise due to model discrepancies, sensing limitations, and the influence of external agents (for example, humans) acting on the system. Stochastic verification can be used to quantify the likelihood of achieving a desired specification with a minimum desired probability, while respecting system dynamics and control bounds [1-3]. A theoretical framework for addressing stochastic reachability and viability problems provides a dynamic programming solution. for the general class of hybrid dynamical systems [4]. However, its numerical implementation relies on gridding, resulting in a lack of scalability. An alternate approach is to pose the stochastic reachability problem as a stochastic optimization problem, and to utilize existing literature on chance constraints [5]. A chance constraints-based approach has been utilized to verify linear systems perturbed by Gaussian disturbance [6-9]. We extend this approach to non-Gaussian disturbances, and propose a Fourier transform-based approach that is tight and sampling-free. We demonstrate that our approach outperforms existing state-of-the-art techniques to enforce chance constraints involving non-Gaussian random vectors.

*This material is based upon work supported by the National Science Foundation under Grant Numbers IIS-1528047, CMMI-1254990, and OIA-1757207. Any opinions, findings, and conclusions or recommendations expressed in this material are those of the authors and do not necessarily reflect the views of the National Science Foundation. ${ }^{\dagger}$ Corresponding author

Permission to make digital or hard copies of all or part of this work for personal or classroom use is granted without fee provided that copies are not made or distributed for profit or commercial advantage and that copies bear this notice and the full citation on the first page. Copyrights for components of this work owned by others than the author(s) must be honored. Abstracting with credit is permitted. To copy otherwise, or republish, to post on servers or to redistribute to lists, requires prior specific permission and/or a fee. Request permissions from Permissions@acm.org.

SNR '19, April 15, 2019, Montreal, QC, Canada

(C) 2019 Copyright is held by the owner/author(s). Publication rights licensed to ACM.

ACM 978-1-4503-6697-7/19/04_..\$15.00

https://doi.org/10.1145/3313149.3313367
We first formally state the problem of interest. Let $\bar{u} \in \mathbb{R}^{n}$ be an optimization variable and $w \in \mathbb{R}^{m}$ be a random vector with probability measure $\mathbb{P}_{\boldsymbol{w}}$. Define the halfspace $\mathcal{H}(\bar{u})=\left\{\bar{w} \in \mathbb{R}^{m}: \bar{a}^{\top} \bar{w} \leq\right.$ $\left.b+\bar{c}^{\top} \bar{u}\right\}$ using known $\bar{a}, b$, and $\bar{c}$ with appropriate dimensions. Given some $\bar{u} \in \mathbb{R}^{n}$ and $p \in(0,1)$, we wish to tractably evaluate the probability of $\boldsymbol{w}$ lying in $\mathcal{H}(\bar{u})$. We denote this probability by $G: \mathbb{R}^{n} \rightarrow[0,1]$,

$$
G(\bar{u}) \triangleq \mathbb{P}_{\boldsymbol{w}}\{\boldsymbol{w} \in \mathcal{H}(\bar{u})\} .
$$

In verification problems, $\bar{u}$ may be the control input to the system, $\boldsymbol{w}$ the disturbance, and $\bar{a}, b$, and $\bar{c}$ may be derived from the polytopic safety constraints, the dynamics of a linear system, and the initial state (see [6] for more details). We also wish to characterize the feasible set for $\bar{u}$, denoted by $\mathcal{F}(p) \subseteq \mathbb{R}^{n}$,

$$
\mathcal{F}(p)=\left\{\bar{u} \in \mathbb{R}^{n}: G(\bar{u}) \geq p\right\} .
$$

The main contributions of this paper are as follows: i) review existing techniques to enforce (1) and characterize (2) for nonGaussian disturbance $\boldsymbol{w}$, and ii) propose a sampling-free approach to enforce the chance constraint using Fourier transforms. We also review known properties about $G(\bar{u})$ and $\mathcal{F}(p)$. Finally, we illustrate the efficacy and accuracy of our method over existing techniques using a numerical example.

\section{REVIEW OF KNOWN RESULTS}

\subsection{Cumulative density function-based reformulation of (1) and (2)}

Let $\Phi_{\bar{a}^{\top} \boldsymbol{w}}(\cdot)$ denote the cumulative density function of the random variable $\bar{a}^{\top} \boldsymbol{w}$. We have

$$
G(\bar{u})=\mathbb{P}_{\boldsymbol{w}}\left\{\bar{a}^{\top} \boldsymbol{w} \leq b+\bar{c}^{\top} \bar{u}\right\}=\Phi_{\bar{a}^{\top} \boldsymbol{w}}\left(b+\bar{c}^{\top} \bar{u}\right)
$$

In addition, we have

$$
\mathcal{F}(p)=\left\{\bar{u} \in \mathbb{R}^{n}: \Phi_{\bar{a}^{\top} \boldsymbol{w}}\left(b+\bar{c}^{\top} \bar{u}\right) \geq p\right\} .
$$

For Gaussian random vectors, $\bar{u} \in \mathcal{F}(p)$ can be enforced exactly as a deterministic linear constraint on $\bar{u}$, using the standard normal cumulative density function [6,7]. The main focus of this paper is to provide a tractable approach to implement (3) and (4) for nonGaussian disturbances.

\subsection{Properties of $G(\bar{u})$ and $\mathcal{F}(p)$}

Lemma 1. [5, Thms. 4.2.4 and 10.2.1] For any probability measure $\mathbb{P}_{\boldsymbol{w}}$, the function $G(\bar{u})$ is upper semicontinuous. For a log-concave probability measure $\mathbb{P}_{\boldsymbol{w}}$, the function $G(\bar{u})$ is log-concave.

Recall that upper semicontinuous functions are functions whose superlevel sets $\mathcal{S}_{\alpha}=\left\{\bar{u} \in \mathbb{R}^{n}: G(\bar{u}) \geq \alpha\right\}$ are closed for every 
$\alpha \in \mathbb{R}$. Log-concavity of $G(\bar{u})$ implies that $\log (G(\bar{u}))$ is concave. Logconcave functions have convex superlevel sets $\mathcal{S}_{\alpha}$ for every $\alpha \in \mathbb{R}$. Note that many standard distributions, like Gaussian, exponential, and beta distributions, have a log-concave probability measure. See [10] for more details.

Remark 1. For a log-concave probability measure $\mathbb{P}_{\boldsymbol{w}}$, the set $\mathcal{F}(p)$ is convex and closed.

Remark 2. The approaches discussed in this paper to compute $G(\bar{u})$ and $\mathcal{F}(p)$ do not require $\mathbb{P}_{\boldsymbol{w}}$ to be a log-concave probability measure.

Recall that the support function of a convex and closed set $\mathcal{S}$ is given by the real-valued function $\rho_{\mathcal{S}}(\bar{\ell}): \mathbb{R}^{n} \rightarrow \mathbb{R}$,

$$
\rho_{\mathcal{S}}(\bar{\ell})=\sup _{\bar{y} \in \mathcal{S}} \bar{\ell}^{\top} \bar{y}
$$

The function $\rho_{\mathcal{S}}(\cdot)$ characterizes the set $\mathcal{S}$ as follows,

$$
\mathcal{S}=\left\{\bar{y} \in \mathbb{R}^{n}: \forall \bar{\ell} \in \mathbb{R}^{n}, \bar{\ell}^{\top} \bar{y} \leq \rho_{\mathcal{S}}(\bar{\ell})\right\} .
$$

Given two convex and closed sets $\mathcal{G}_{1}, \mathcal{G}_{2} \subseteq \mathbb{R}^{n}$, then

$$
\mathcal{G}_{1} \subseteq \mathcal{G}_{2} \text { if and only if } \rho_{\mathcal{G}_{1}}(\bar{\ell}) \leq \rho_{\mathcal{G}_{2}}(\bar{\ell}), \forall \ell \in \mathbb{R}^{n} .
$$

See [10, Sec. 7.1.2] for more details.

\subsection{Moment-based restriction}

Concentration inequalities provide several useful probability bounds $[11,12]$. As discussed in [12], one can use Cantelli-Chebyshev inequality to obtain a sampling-free restriction of $\mathcal{F}(p)$ for any $p \in(0,1)$. From Cantelli-Chebyshev inequality,

$$
G(\bar{u}) \geq 1-\frac{\bar{a}^{\top} \Sigma_{\boldsymbol{w}} \bar{a}}{\bar{a}^{\top} \sum_{\boldsymbol{w}} \bar{a}+\left(b+\bar{c}^{\top} \bar{u}-\bar{a}^{\top} \mu_{\boldsymbol{w}}\right)^{2}} .
$$

By (8), we have $\mathcal{F}_{\mathrm{m}}(p) \subseteq \mathcal{F}(p) \subseteq \mathbb{R}^{n}$,

$$
\mathcal{F}_{\mathrm{m}}(p) \triangleq\{\bar{u}:-\bar{c}^{\top} \bar{u} \leq \underbrace{b-\bar{a}^{\top} \bar{\mu}_{\boldsymbol{w}}-\sqrt{\frac{p\left(\bar{a}^{\top} \Sigma_{\boldsymbol{w}} \bar{a}\right)}{1-p}}}_{d}\} .
$$

Equation (9) is a linear constraint on $\bar{u}$. A comparison of the bounds (4) and (9) for a Gaussian $\boldsymbol{w}$ is given in [13, Fig. 2].

Proposition 1. If the set $\mathcal{F}(p)$ is convex and closed, then $\mathcal{F}(p)$ is a halfspace for every $p \in(0,1)$.

Proof: By (7) and (9), we have for every $\bar{\ell} \in \mathbb{R}^{n}$

$$
\rho_{\mathcal{F}(p)}(\bar{\ell}) \geq \rho_{\mathcal{F}_{\mathrm{m}}(p)}(\bar{\ell})=\left\{\begin{array}{lr}
\lambda d, & \exists \lambda \in \mathbb{R}_{\geq 0} \text { s. t. } \lambda \bar{c}+\bar{\ell}=0 \\
+\infty, & \text { otherwise }
\end{array}\right.
$$

Here, (10) follows from strong duality of linear programming. Let $d^{+} \triangleq \rho_{\mathcal{F}(p)}(-\bar{c}) \geq d$. By $(6)$ and $(10), \mathcal{F}(p)$ can be written as

$$
\mathcal{F}(p)=\left\{\bar{u} \in \mathbb{R}^{n}:-\bar{c}^{\top} \bar{u} \leq d^{+}\right\} .
$$

This completes the proof.

\subsection{Sample average approximation}

Sample average approximation (SAA) uses realizations of $\boldsymbol{w}$ to enforce the chance constraint (2) [10, Ch. 5]. We will denote samples of $\boldsymbol{w}$ as $\bar{w}^{(\cdot)}$. For any $p \in(0,1)$ and $N \in \mathbb{N}$ samples of $\boldsymbol{w}$, we can approximately evaluate $G(\bar{u})$ and enforce $\mathcal{F}(p)$ approximately using $\mathcal{F}_{\mathrm{s}}(p)$ as follows,

$$
\begin{aligned}
& G(\bar{u}) \approx \frac{1}{N} \sum_{i=1}^{N} 1_{[0, \infty)}\left(b+\bar{c}^{\top} \bar{u}-\bar{a}^{\top} \bar{w}^{(i)}\right) \\
& \mathcal{F}_{\mathrm{S}}(p) \triangleq\left\{\bar{u} \in \mathbb{R}^{n}: \sum_{i=1}^{N} 1_{[0, \infty)}\left(b+\bar{c}^{\top} \bar{u}-\bar{a}^{\top} \bar{w}^{(i)}\right) \geq p N\right\}
\end{aligned}
$$

Here, $1_{[0, \infty)}(z)=1$ if $z \geq 0$ and 0 otherwise. The approximation error in (12) converges to zero as $N \rightarrow \infty$ [10, Prop. 5.1]. Recently, lower bounds for $N$ that provide probabilistic guarantees on the approximation error have been proposed. However, these lower bounds typically require large $N$, which results in significant conservativeness [10, Ch. 5].

\subsection{Conditional Value-at-Risk-based restriction}

Recall the following observations:

1) $\mathbb{P}_{z}\{z \geq 0\}=\mathbb{E}_{z}\left[1_{[0, \infty)}(z)\right]$ for any random variable $z$, and

2) $\max \left(0, \frac{z}{\alpha}+1\right) \geq 1_{[0, \infty)}(z)$ for every $z \in \mathbb{R}$ and $\alpha>0$.

Let $z \triangleq \bar{a}^{\top} \boldsymbol{w}-b-\bar{c}^{\top} \bar{u}$ with $\mathbb{P}_{\boldsymbol{z}}\{\boldsymbol{z}=0\}=0$. By properties of expectations and above observations,

$$
G(\bar{u})=1-\mathbb{P}_{\boldsymbol{z}}\{\boldsymbol{z} \geq 0\} \geq 1-\mathbb{E}_{\boldsymbol{w}}\left[\max \left(0, \frac{\bar{a}^{\top} \boldsymbol{w}-b-\bar{c}^{\top} \bar{u}}{\alpha}+1\right)\right]
$$

for any $\alpha>0$. Note that the right-hand side of (13) is a concave function in $\bar{u}$, and minimizing $\alpha$ for the tightest lower bound provides the Conditional Value-at-Risk (CVaR) bound on $G(\bar{u})[14,15]$. The inequality in (13) provides an inner approximation $\mathcal{F}_{\text {cvar }}(p) \subseteq$ $\mathcal{F}(p) \subseteq \mathbb{R}^{n}$ for any $p \in(0,1)$,

$$
\mathcal{F}_{\text {cvar }}=\left\{\bar{u}: \mathbb{E}_{\boldsymbol{w}}\left[\max \left(0, \frac{\bar{a}^{\top} \boldsymbol{w}-b-\bar{c}^{\top} \bar{u}}{\alpha}+1\right)\right] \leq 1-p\right\} .
$$

For an arbitrary random vector $\boldsymbol{w}$, we typically do not have a closedform expression for the expectation in (14). A common approach to implement (13) and (14) is to use SAA as done in Section 2.4.

\section{FOURIER TRANSFORMS-BASED APPROACH}

The characteristic function of a random vector $\boldsymbol{w} \in \mathbb{R}^{m}$ with probability density function $\psi_{\boldsymbol{w}}(\bar{z})$ is

$$
\begin{aligned}
\Psi_{\boldsymbol{w}}(\bar{\beta}) & \triangleq \mathbb{E}_{\boldsymbol{w}}\left[\exp \left(j \bar{\beta}^{\top} \boldsymbol{w}\right)\right] \\
& =\int_{\mathbb{R}^{p}} e^{j \bar{\beta}^{\top} \bar{z}} \psi_{\boldsymbol{w}}(\bar{z}) d \bar{z}=\mathscr{F}\left\{\psi_{\boldsymbol{w}}(\cdot)\right\}(-\bar{\beta})
\end{aligned}
$$

where $\mathscr{F}\{\cdot\}$ denotes the Fourier transformation operator and $\bar{\beta} \in$ $\mathbb{R}^{m}$. Note that characteristic functions of standard distributions are well studied and have closed-form expressions [16]. From [17, Prop. P2] (or, see [16, Eq. 22.6.3]), the characteristic function of the random variable $\bar{a}^{\top} \boldsymbol{w}$ is given by,

$$
\Psi_{\bar{a}^{\top} \boldsymbol{w}}(t)=\Psi_{\boldsymbol{w}}(t \bar{a}), \text { for } t \in \mathbb{R} .
$$




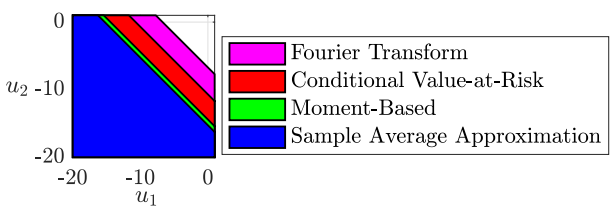

Figure 1: Approximations of $\mathcal{F}(p)$ via various approaches. All the sets are halfspaces by Proposition 1.

We can compute (3) directly by the following well-known inversion of $\Psi_{\bar{a}^{\top} \boldsymbol{w}}$ defined in (16) [18-20]

$$
G(\bar{u})=\frac{1}{2}-\frac{1}{2 \pi} \int_{-\infty}^{\infty} \operatorname{Im}\left(\frac{e^{\left(-j t\left(b+\bar{c}^{\top} \bar{u}\right)\right)} \Psi_{w}(t \bar{a})}{j t}\right) d t
$$

where $\operatorname{Im}(\cdot)$ denotes the imaginary component of the evaluation of a complex function. We can implement (17) using quadrature techniques [21]. Further, using (11), we can also compute $\mathcal{F}(p)$.

\subsection{Advantages of this approach over others}

Recall that the characteristic function $\Psi_{\bar{a}^{\top} \boldsymbol{w}}(t)$ characterizes all the moments of $\bar{a}^{\top} \boldsymbol{w}$. Thus, the Fourier transform-based (FT) approach computes $G(\bar{u})$ in (17) using all the moments of $\bar{a}^{\top} \boldsymbol{w}$. In contrast, the moment-based approach (8) uses information only up to second moment. The conservativeness of the $\mathrm{CVaR}$ approach with respect to the FT approach can be seen from (13) and (17). Due to its reliance on sampling, the SAA approach (12a) converges to the FT approach (17) only asymptotically.

\section{NUMERICAL EXAMPLE}

We use SReachTools [22] to set up the problems, CharFunTool for the proposed FT approach [21], and CVX [23] for the CVaR approach. We presume $\boldsymbol{w}$ as a two-dimensional exponential random vector with mean $\mu_{\boldsymbol{w}}=\left[\begin{array}{ll}2 & 4\end{array}\right]^{\top}$, and that $\bar{a}=\left[\begin{array}{ll}1 & 1\end{array}\right]^{\top}, b=5$, and $\bar{c}=-\left[\begin{array}{ll}1 & 1\end{array}\right]^{\top}$.

Since the exponential distribution is $\log$-concave, $\mathcal{F}(p)$ is a halfspace, by Proposition 1. Figure 1 shows the approximations of $\mathcal{F}(p)$ characterized by (11). Since our proposed method (FT) directly computes $\Phi_{\bar{a}^{\top} \boldsymbol{w}}(\cdot)$ using (17), it contains the approximations of $\mathcal{F}(p)$ from all of the other methods. The only other sampling-free technique, the moment-based set (9), is significantly more conservative.

Figure 2 shows the quality of approximation of $G(\bar{u})$ as well as the computation time for each of the method. The maximum variance in $G(\bar{u})$ for SAA (12a), CVaR (13), and FT (17) were $5.80 \times 10^{-3}$, $1.40 \times 10^{-3}$, and $1.24 \times 10^{-16}$ respectively for the varying number of samples (in case of SAA and CVaR) or points of integration (in case of FT). We compute the moment-based bound (8) directly.

\section{CONCLUSION}

This paper reviews the current state-of-the-art techniques to enforce individual chance constraints involving non-Gaussian random vectors. We also propose a Fourier transform-based sampling-free approach that outperforms all of the existing approaches.

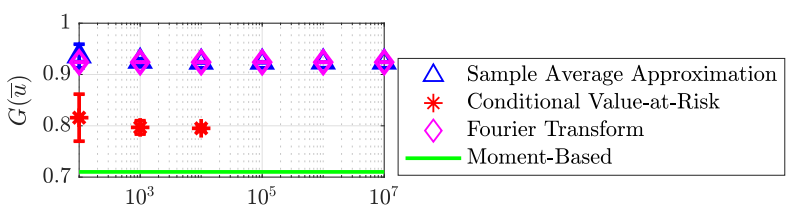

Number of Points for Integration (FT) or Samples (SAA/CVaR)

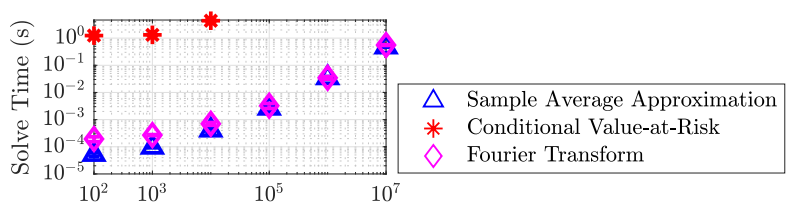

Number of Points for Integration (FT) or Samples (SAA/CVaR)

Figure 2: Comparison of $G(\bar{u})$ and compute times.

\section{REFERENCES}

[1] A. Abate, M. Prandini, J. Lygeros, and S. Sastry. Probabilistic reachability and safety for controlled discrete time stochastic hybrid systems. Automatica, 44(11):2724-2734, 2008.

[2] S. Summers and J. Lygeros. Verification of discrete time stochastic hybrid systems: A stochastic reach-avoid decision problem. Automatica, 46(12):1951-1961, 2010.

[3] A. Vinod and M. Oishi. Stochastic reachability of a target tube: Theory and computation. IEEE Trans. Auto. Ctrl., 2018. (submitted) https://arxiv.org/abs/1810. 05217.

[4] A. Abate, S. Amin, M. Prandini, J. Lygeros, and S. Sastry. Computational approaches to reachability analysis of stochastic hybrid systems. In Proc. Hybrid Syst.: Comput. and Ctrl., pages 4-17, 2007.

[5] A. Prékopa. Stochastic programming. Springer Science \& Business Media, 1995.

[6] K. Lesser, M. Oishi, and R. Erwin. Stochastic reachability for control of spacecraft relative motion. In IEEE Conf. Dec. Ctrl., pages 4705-4712, 2013.

[7] M. Ono and B. Williams. Iterative risk allocation: A new approach to robust model predictive control with a joint chance constraint. In IEEE Conf. Dec. Ctrl., pages 3427-3432, 2008.

[8] F. Oldewurtel, C. Jones, A. Parisio, and M. Morari. Stochastic model predictive control for building climate control. IEEE Trans. Ctrl. Syst. Tech., 22(3):1198-1205, 2014.

[9] M. Vitus and C. Tomlin. On feedback design and risk allocation in chance constrained control. In IEEE Conf. Dec. Ctrl., pages 734-739, 2011.

[10] A. Shapiro, D. Dentcheva, and A. Ruszczyński. Lectures on stochastic programming: modeling and theory. SIAM, 2009.

[11] O. Bouissou, E. Goubault, S. Putot, A. Chakarov, and S. Sankaranarayanan. Uncertainty propagation using probabilistic affine forms and concentration of measure inequalities. In Int'l Conf. Tools and Algorithms for the Construction and Analysis of Systems, pages 225-243, 2016.

[12] J. Paulson, E. Buehler, R. Braatz, and A. Mesbah. Stochastic model predictive control with joint chance constraints. Int'l f. Control, pages 1-14, 2017.

[13] M. Farina, L. Giulioni, and R. Scattolini. Stochastic linear model predictive control with chance constraints-a review. F. Process Ctrl., 44:53-67, 2016.

[14] A. Nemirovski and A. Shapiro. Convex approximations of chance constrained programs. F. Optimization, 17(4):969-996, 2006.

[15] M. Vitus. Stochastic control via chance constrained optimization and its application to unmanned aerial vehicles. PhD thesis, Stanford University, 2012.

[16] H. Cramér. Mathematical methods of statistics. Princeton Univ. Press, 1961.

[17] A. Vinod, B. HomChaudhuri, and M. Oishi. Forward stochastic reachability analysis for uncontrolled linear systems using Fourier transforms. In Proc. Hybrid Syst.: Comput. and Ctrl., pages 35-44, 2017.

[18] J Gil-Pelaez. Note on the inversion theorem. Biometrika, 38(3-4):481-482, 1951.

[19] L. Waller, B. Turnbull, and J. Hardin. Obtaining distribution functions by numerical inversion of characteristic functions with applications. The American Statistician, 49(4):346-350, 1995.

[20] V. Witkovský. Numerical inversion of a characteristic function: An alternative tool to form the probability distribution of output quantity in linear measurement models. Acta IMEKO, 5(3):32-44, 2016.

[21] V. Witkovský. CharFunTool: The characteristic functions toolbox. https://github. com/witkovsky/CharFunTool.

[22] A. Vinod, J. Gleason, and M. Oishi. SReachTools: Stochastic reachability toolbox for MATLAB. In Proc. Hybrid Syst.: Comput. and Ctrl., 2019. https://unm-hscl. github.io/SReachTools (accepted).

[23] M. Grant and S. Boyd. CVX: Matlab software for disciplined convex programming, version 2.1. http://cvxr.com/cvx, March 2014. 